\title{
Effect of high-intensity ultrasound specific energy on the bixin and geranylgeraniol recovery from defatted annatto seeds
}

\section{Gabriela F. Freitas*, Monique M. Strieder, Renata Vardanega, M. Angela A. Meireles, Eric Keven Silva}

\begin{abstract}
Annatto (Bixa orellana L.) seeds are rich in bioactive compounds as bixin, an important carotenoid used as a colorant, and geranylgeraniol, a terpenic compound which presents several biological activities as apoptosis of carcinogen cells. The extraction of these bioactive compounds using clean emerging technology and a green solvent like ethanol can boost their health benefit effects. In this sense, the aim of this study was to evaluate the influence of high-intensity ultrasound (HIUS) specific energy on the extraction of bixin and geranylgeraniol from defatted annatto seeds. The extractions were performed using two HIUS processes, low power and long time (LPLT) and high power and short time (HPST); both operating at the same specific energy levels $(1,3,5$, and $7 \mathrm{~kJ} / \mathrm{g}$ ). The most effective extraction was obtained by using the HPST process and the specific energy of $7 \mathrm{~kJ} / \mathrm{g}$, which allowed to obtain a higher global yield (8.2 $\mathrm{g} / 100 \mathrm{~g}$ annatto seeds). In addition, was verified that the HIUS-assisted extraction did not modify the colorant's color parameters and solubility. However, there were morphological changes as the specific energy increased.
\end{abstract}

\section{Key words:}

IEmerging technology, bioactive compounds, green solvent.

\section{Introduction}

The annatto processing chain is responsible for the production of bixin, an important natural colorant used by the food, pharmaceutical, and cosmetic industries. In addition, recently the annatto seeds were reported as a new source of geranylgeraniol, a bioactive compound with several biological activities, as therapeutic action against Chagas disease and apoptosis of carcinogen cells. In this sense, the processing of this vegetable matrix using a biorefinary approach based on emerging technologies, like supercritical carbon dioxide technology (SCDT) for the extraction of lipophilic bioactive compounds followed by the HIUS technology for the colorant recovery, presents highpotential for production of health-promoter compounds and well-being. Therefore, the aim of this study was to evaluate the effects of HIUS technology on the recovery of bixin and geranylgeraniol from defatted annatto seeds after oil extraction step using SCDT. The HIUS processing was performed using two ways: low power and long time (LPLT) and high power and short time (HPST); both operating at the same specific energy levels $(1,3,5$, and $7 \mathrm{~kJ} / \mathrm{g})$.

\section{Results and Discussion}

For the LPLT process using a nominal power of $100 \mathrm{~W}$, the processing times were $5,15,25$ and $35 \mathrm{~min}$; and for the HPST process using $475 \mathrm{~W}$, were $1.05,3.15,5.25$, and $7.37 \mathrm{~min}$. Figure 1 shows the global yields of these processes. Higher yields were obtained by the HPST process. Figure 2 and 3 show the results of bixin and geranylgeraniol extraction yields, respectively. The increase of HIUS specific energy resulted in higher extraction yields due to process intensification. The characterizations performed by ultraviolet-visible and Fourier-transform spectroscopy showed that there were no changes in the structure and composition of functional groups of all HIUS extracts. Color parameters and solubility of the colorant were not influenced by HIUS specific energy; however, its morphological characteristics were modified with the increase the specific energy. This effect can be associated with the cavitation phenomenon, which is responsible for breaking down the cell wall, resulting in small fragmentations. However, those ruptures did not affect any other physicochemical properties of the colorants.

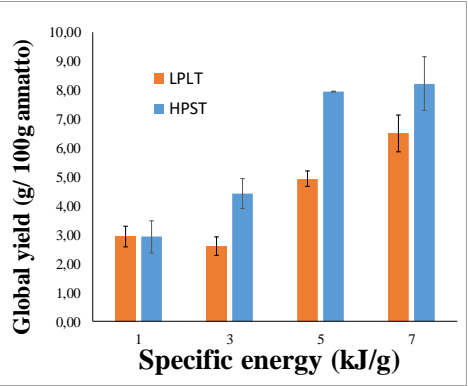

Figure 1. Global extraction yields for the LPLT and HPST processes at the specific energies of $1,3,5$ and $7 \mathrm{~kJ} / \mathrm{g}$.

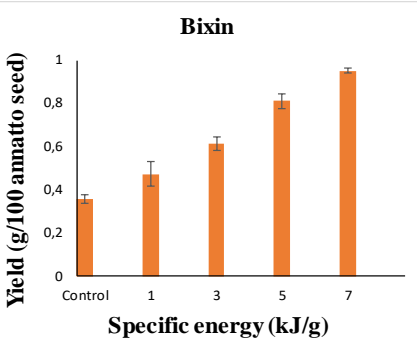

Figure 2. Bixin yields.

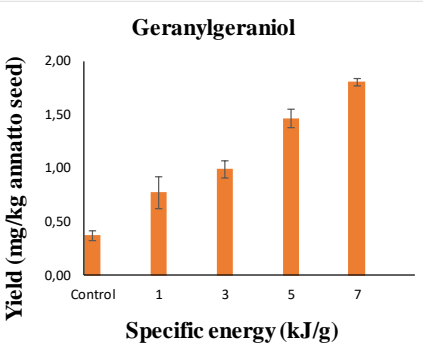

Figure 3. Geranylgeraniol yields.

\section{Conclusions}

The HPST process using $7 \mathrm{~kJ} / \mathrm{g}$ was the most efficient HIUS treatment to extract the natural colorant from annatto seeds. In this experimental condition was obtained a yield of $0.95 \mathrm{~g}$ bixin $/ 100 \mathrm{~g}$ annatto seed and $1.80 \mathrm{mg}$ geranylgeraniol $/ \mathrm{kg}$ annatto seed. In addition, color parameters and solubility remained unchanged after the HIUS-assisted extraction.

\section{Acknowledgement}

Gabriela F. Freitas thanks SAE-UNICAMP. Monique M. Strieder thanks CNPq (141110/2018-0). Renata Vardanega thanks CAPES (Financial Code - 001). M. Angela A. Meireles thanks CNPq (302423/2015-0). Eric Keven Silva thanks FAPESP (2015/22226-6). 